\title{
Development and evaluation of a light emitting diode endoscopic light source
}

\author{
Neil T. Clancy* ${ }^{1,2}$, Rui Li ${ }^{1,2}$, Kevin Rogers ${ }^{3,4}$, Paul Driscoll ${ }^{4}$, Peter Excel ${ }^{5}$, Ron Yandle ${ }^{4}$, George \\ Hanna $^{2}$, Nigel Copner ${ }^{3,4}$, Daniel S. Elson ${ }^{1,2}$ \\ ${ }^{1}$ Hamlyn Centre for Robotic Surgery, Imperial College London, UK; \\ ${ }^{2}$ Department of Surgery and Cancer, Imperial College London, UK; \\ ${ }^{3}$ Faculty of Advanced Technology, University of Glamorgan, UK; \\ ${ }^{4}$ Cymtec Ltd. Cardiff, UK; \\ ${ }^{5}$ Glyndwr University, Wrexham, UK
}

\begin{abstract}
Light-emitting diode (LED) based endoscopic illumination devices have been shown to have several benefits over arclamp systems. LEDs are energy-efficient, small, durable, and inexpensive, however their use in endoscopy has been limited by the difficulty in efficiently coupling enough light into the endoscopic light cable. We have demonstrated a highly homogenised lightpipe LED light source that combines the light from four Luminus LEDs emitting in the red, green, blue and violet using innovative dichroics that maximise light throughput. The light source spectrally combines light from highly divergent incoherent sources that have a Lambertian intensity profile to provide illumination matched to the acceptance numerical aperture of a liquid light guide or fibre bundle. The LED light source was coupled to a standard laparoscope and performance parameters (power, luminance, colour temperature) compared to a xenon lamp. Although the total illuminance from the endoscope was lower, adjustment of the LEDs' relative intensities enabled contrast enhancement in biological tissue imaging. The LED light engine was also evaluated in a minimally invasive surgery (MIS) box trainer and in vivo during a porcine MIS procedure where it was used to generate 'narrowband' images. Future work using the violet LED could enable photodynamic diagnosis of bladder cancer.
\end{abstract}

Keywords: Light-emitting diode (LED), laparoscopy, minimally-invasive surgery

\section{INTRODUCTION}

Minimally-invasive surgery (MIS) has enabled diagnosis and intervention for patients in a manner that minimizes trauma and reduces recovery time. During these procedures the surgeon does not have a direct line-of-sight to the area under investigation and is therefore dependent on the quality of the imaging system to relay information on the health of the tissue as well as its general appearance. Due to the high attenuation of visible light in biological tissue and the relatively poor light coupling ability of endoscopes, the quality of the light delivery system is of utmost importance.

The current standard light source for most MIS procedures, particularly laparoscopy, is the xenon arc lamp, which emits over a broad spectrum across the visible range, providing a colour close to daylight. These lamps are high power but highly inefficient, requiring approximately $300 \mathrm{~W}$ of electrical power to deliver just over $1 \mathrm{~W}$ of optical power. They are also expensive, requiring an initial payment of about $\$ 8000$ and $\$ 1500$ for replacement bulbs, which have a relatively short lifetime.

Alternative sources such as laser-phosphor fibres and supercontinuum lasers have been explored but these have tended to focus on niche areas that require specialized lighting such as narrow lumen work and the single access surgery regime $^{1}$ where high optical power per cross-sectional area is required. A more significant limitation is perhaps the fact that these devices are currently limited to research models. Commercial devices have focused on providing the surgeon with more information by enhancing contrast in the tissue. This has been achieved using fluorescence to aid visualization of tumours ${ }^{2}$, and narrowband imaging (NBI) to increase the visibility of the vasculature ${ }^{3}$. However, separate systems are required for each of these approaches, leading to increased expenditure. Previous work has indicated that tip-mounted

*n.clancy@imperial.ac.uk; imperial.ac.uk/roboticsurgery

Advanced Biomedical and Clinical Diagnostic Systems X, edited by Tuan Vo-Dinh

Anita Mahadevan-Jansen, Warren S. Grundfest, Proc. of SPIE Vol. 8214, 82140R - (c) 2012 SPIE

CCC code: $1605-7422 / 12 / \$ 18 \cdot$ doi: $10.1117 / 12.909331$

Proc. of SPIE Vol. 821482140 R-1 
LEDs have advantages over the standard arc-lighting systems in terms of uniformity of illumination, stability and visual perception but did generate a large amount of heat ${ }^{4}$.

In this paper a new LED light source is described that uses four ultrabright LEDs in the red, green, blue and violet $(\lambda<430 \mathrm{~nm})$ spectral regions whose relative intensities may be adjusted to provide varying levels of white or coloured illumination. It is capable of generating white light at a similar imaging quality to the standard xenon sources, blue light for excitation of fluorophores in photodynamic diagnosis, and an NBI modality when used with the known spectral properties of the imaging RGB camera. Since it uses LEDs, it is efficient, long-life and inexpensive.

\section{MATERIALS AND METHODS}

\subsection{LED light engine}

The LED light source is shown in Figure 1. Each of the red, green and blue LEDs is controlled by a switch and graduated 10-turn dial, which may be locked to maintain a particular setting. The violet LED is adjusted by a 6-notch dial. The light pipe multiplexer is shown in Figure 1 (b) and is optimized to maximize light collection from the divergent LEDs. Light from the light pipe is coupled into an endoscope light cable $(5 \mathrm{~mm}$ diameter optical fibre bundle; Karl Storz GmbH, Germany).
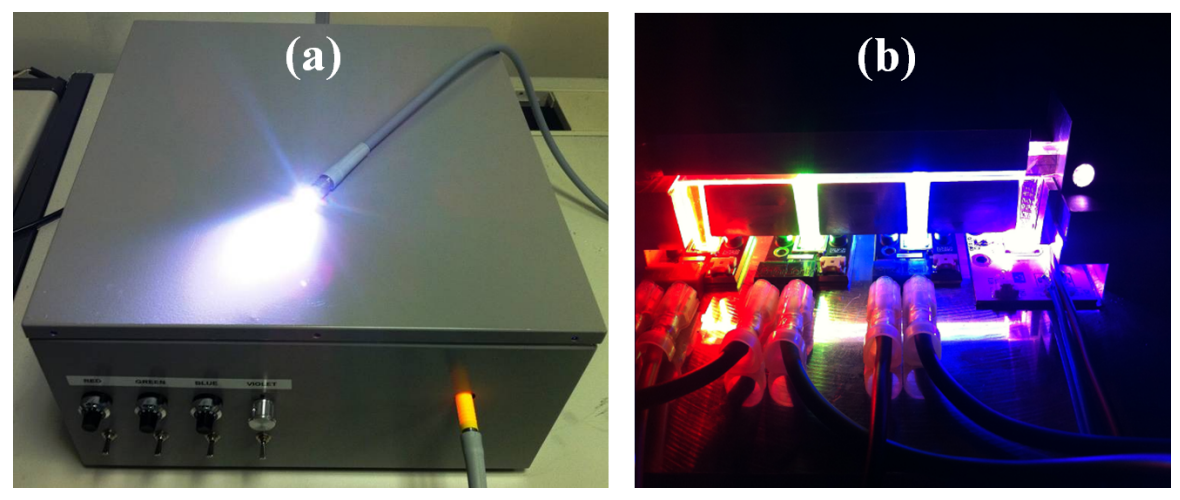

Figure 1. The Cymtec LED light source. (a) The front panel controls adjust the intensity of the four Luminus LEDs. (b)

Light pipe multiplexer to combine light from each of the four LEDs and couple it into the light cable.

\subsection{Comparison with xenon lamp}

The LED light source was compared to a xenon arc lamp (Telepack, Karl Storz GmbH, Germany) on the basis of output power and colour. Output power (illuminance) at the distal end of the light cable was measured using a broadband thermopile power meter (PM10, Coherent, Inc., USA) placed $10 \mathrm{~mm}$ from the source. The spectral output of the light source was measured using a spectrometer (HR4000, Ocean Optics, Inc., USA) which was corrected for wavelengthdependent variations in sensitivity using a calibrated light source (LS-1Cal, Ocean Optics, Inc., USA). The chromaticity coordinates of the colour output of the LED source were calculated by integrating the emission spectrum for a particular combination of LED settings under the CIE 1931 tristimulus curves ${ }^{5}$. Illuminance and chromaticity were measured for a range of LED settings and combinations.

For a qualitative comparison, both light sources were used to image artificial organs in a laparoscopy abdominal simulator $^{6}$, ex vivo tissue (lamb's kidney, liver) and in vivo during a porcine trial (conducted under UK home office approved license PPL 80/2297). Images were acquired with the LED in 'white light' mode as well as predetermined settings that resulted in colours tracing the Planckian chromaticity locus and other combinations of the base LED spectra. The camera was automatically white-balanced using a white sheet of paper prior to imaging using each source and the automatic exposure time mode was used.

\subsection{Colour balance and contrast enhancement}

Optical properties of biological tissue vary widely depending on the tissue type and may be highly reflective (fat), highly absorptive of blue/green light (blood) or may have fluorescent properties (collagen). Selecting an appropriate wavelength 
range to interrogate the tissue with can allow contrast to be enhanced for specific surgical tasks such as visualizing the vasculature. Current surgical light sources usually require the incorporation of various excitation filters in order to attenuate unwanted wavelengths in the source, or emission filters placed on the camera side to detect only the spectral region of interest. In this paper, the emission properties of the LED light sources are tuned by adjusting the power of the constituent red, green, blue and violet elements.

\section{RESULTS}

\subsection{Comparison with xenon lamp}

The output spectra of the LED source and xenon lamp are shown in Figure 2 (a). The characteristic xenon peaks are visible while the relative emission strengths of the four LEDs are also evident. The maximum power achieved using the xenon and LED sources were $1.50 \mathrm{~W}$ and $0.61 \mathrm{~W}$ respectively. Adjusting the relative intensities of the LEDs in the unit made it possible to obtain different colour temperatures, which are plotted on the CIE 1931 chromaticity diagram in Figure 2 (b). It can be seen that the broad spectrum xenon lamp results in an output colour very close to natural daylight, but also that the LED source is capable of achieving a similar result. The LED settings used to achieve particular output colours are given in Table 1.

Table 1. Relative LED intensities used to obtain specific colour temperatures (CCT) and colours.

\begin{tabular}{ccccc|ccccc}
\hline CCT / K & Red & Green & Blue & Violet & Colour & Red & Green & Blue & Violet \\
\hline 6500 & 10.00 & 10.00 & 10.00 & off & 'Red' & 10.00 & off & off & off \\
6000 & 7.60 & 6.90 & 10.00 & off & 'Green' & off & 10.00 & off & off \\
5500 & 9.70 & 7.90 & 10.00 & off & 'Blue' & off & off & 10.00 & off \\
5000 & 10.00 & 7.00 & 9.00 & off & 'Violet' & off & off & off & full \\
4500 & 10.00 & 7.00 & 5.70 & off & 'Yellow' & 10.00 & 10.00 & off & off \\
4000 & 10.00 & 6.10 & 4.00 & off & 'Purple' & 10.00 & off & 10.00 & off \\
3500 & 10.00 & 6.10 & 4.00 & off & $\begin{array}{c}\text { 'Green- } \\
\text { Blue' }\end{array}$ & off & 10.00 & 10.00 & off \\
3000 & 7.30 & 2.80 & 1.90 & off & 'full & 10.00 & 10.00 & 10.00 & full \\
2500 & 5.80 & 2.05 & 0.80 & off & & & & & \\
\hline
\end{tabular}

Qualitative images of the tissue phantoms and ex vivo tissue are shown in Figure 3. After white balancing, the LEDs produce an image that is qualitatively very similar to those obtained using the xenon source albeit slightly redder overall. The LED source allows visualization of the large cavity of the simulated abdomen equally as well as the xenon lamp. There is no noticeable difference in noise between the images acquired with both sources despite the difference in power, and exposure times used were of the same order (tens of milliseconds). 

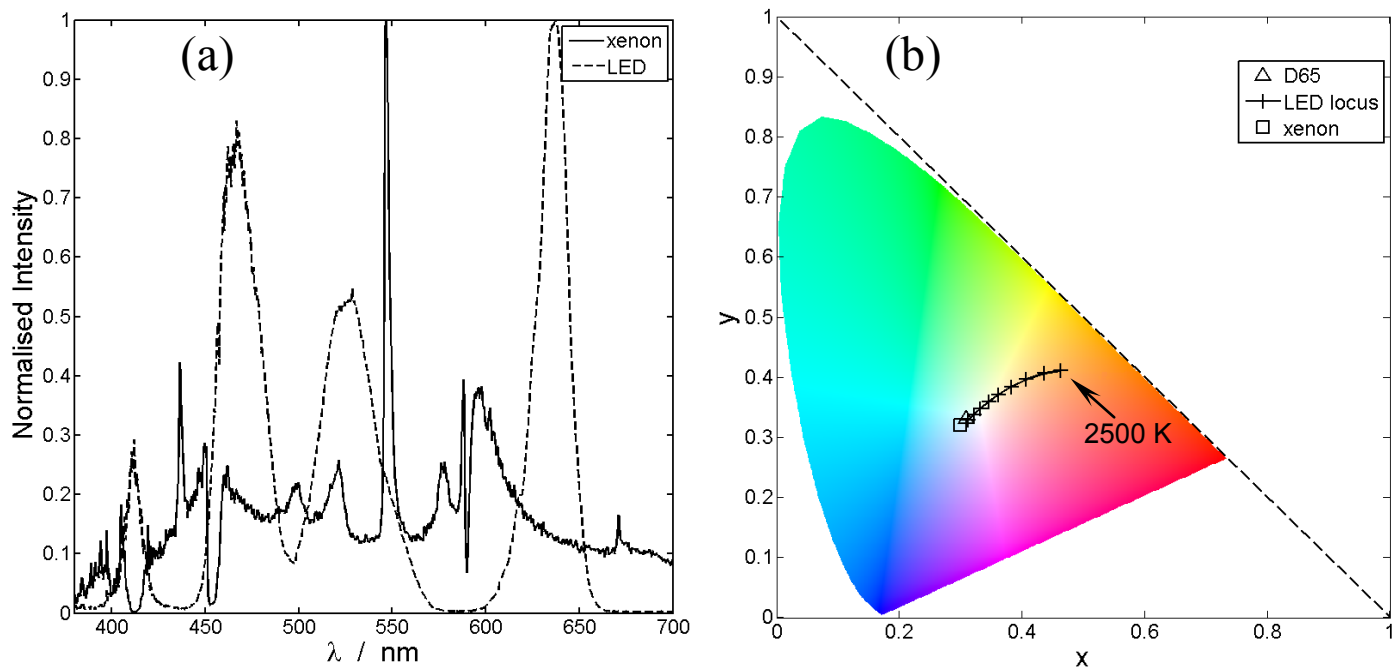

Figure 2. (a) Emission spectra of the xenon lamp (solid line) and LED light source (dashed) in the visible range. (b)

Chromaticity diagram showing the colour coordinates of the standard D65 illuminant ('daylight'), xenon lamp and a range of values along the Planckian locus obtained by tuning the relative LED intensities.

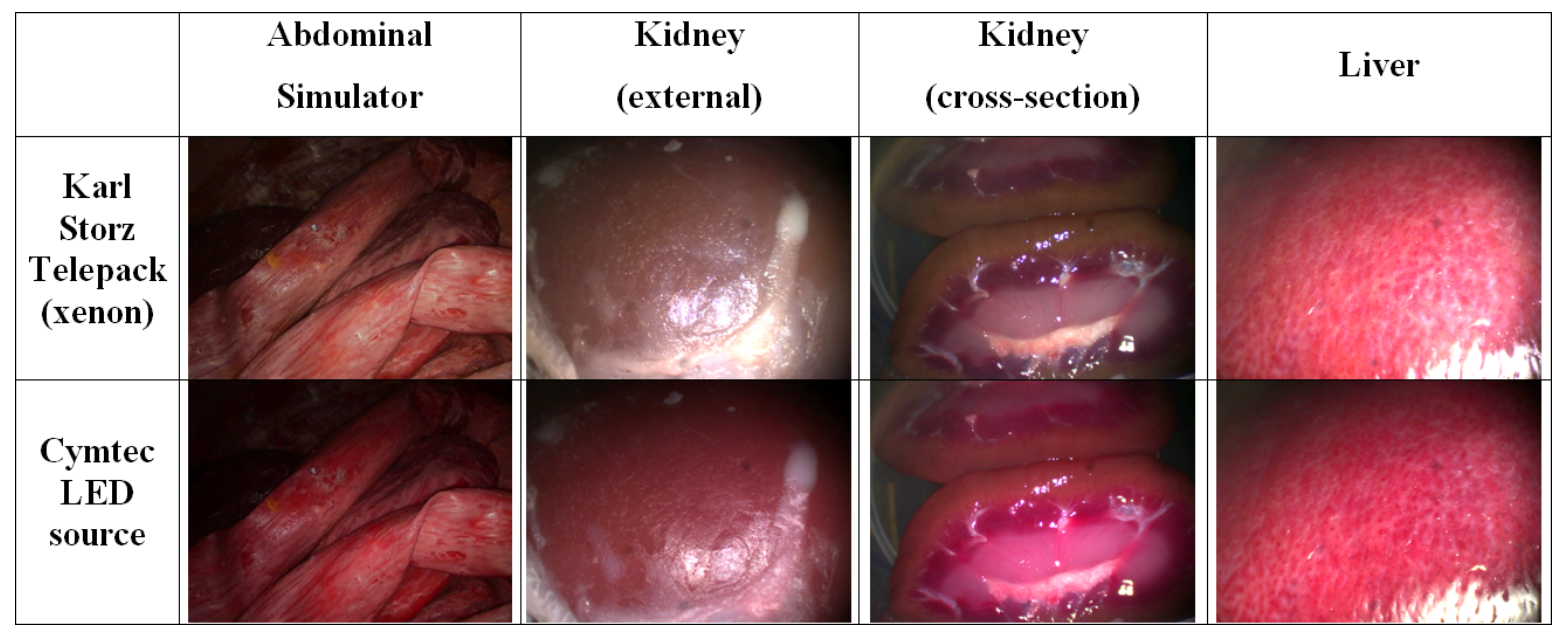

Figure 3. Qualitative comparison of xenon and LED illumination with LED source set for maximum output power.

\subsection{Colour balance and contrast enhancement}

Figure 4 shows ex vivo tissues of varying optical properties under LED illumination of varying colours as defined in Table 1. The lowest contrast images are those under red illumination due to the relatively low absorption coefficient of haemoglobin in this region. The fat is visible as being more reflective than the background tissue but the renal pyramids in the kidney are not easily distinguished and the surface of the liver appears relatively homogeneous. Green and blue show better contrast as blood absorbs more strongly at these wavelengths compared to fatty tissue. The structures inside the kidney are more defined and the patterning on the liver's surface is clearly visible. The violet LED is most strongly absorbed and is also the least bright LED leading to very low intensity images, even at longer exposure times (approaching $1 \mathrm{~s}$ ).

The visibility of the tissue structures is increased by using combinations of the LEDs. 'Yellow' and 'green-blue' illumination each show increased contrast over the single LED images. However, the green-blue appears to provide superior contrast to both of the other two or white light imaging as the fine structures running from the cortex of the kidney to the renal pyramids are more visible. In this case, the presence of red light provides a background signal that penetrates to a similar degree through all of the tissue structures, drowning out the signal from smaller features and reducing contrast. 

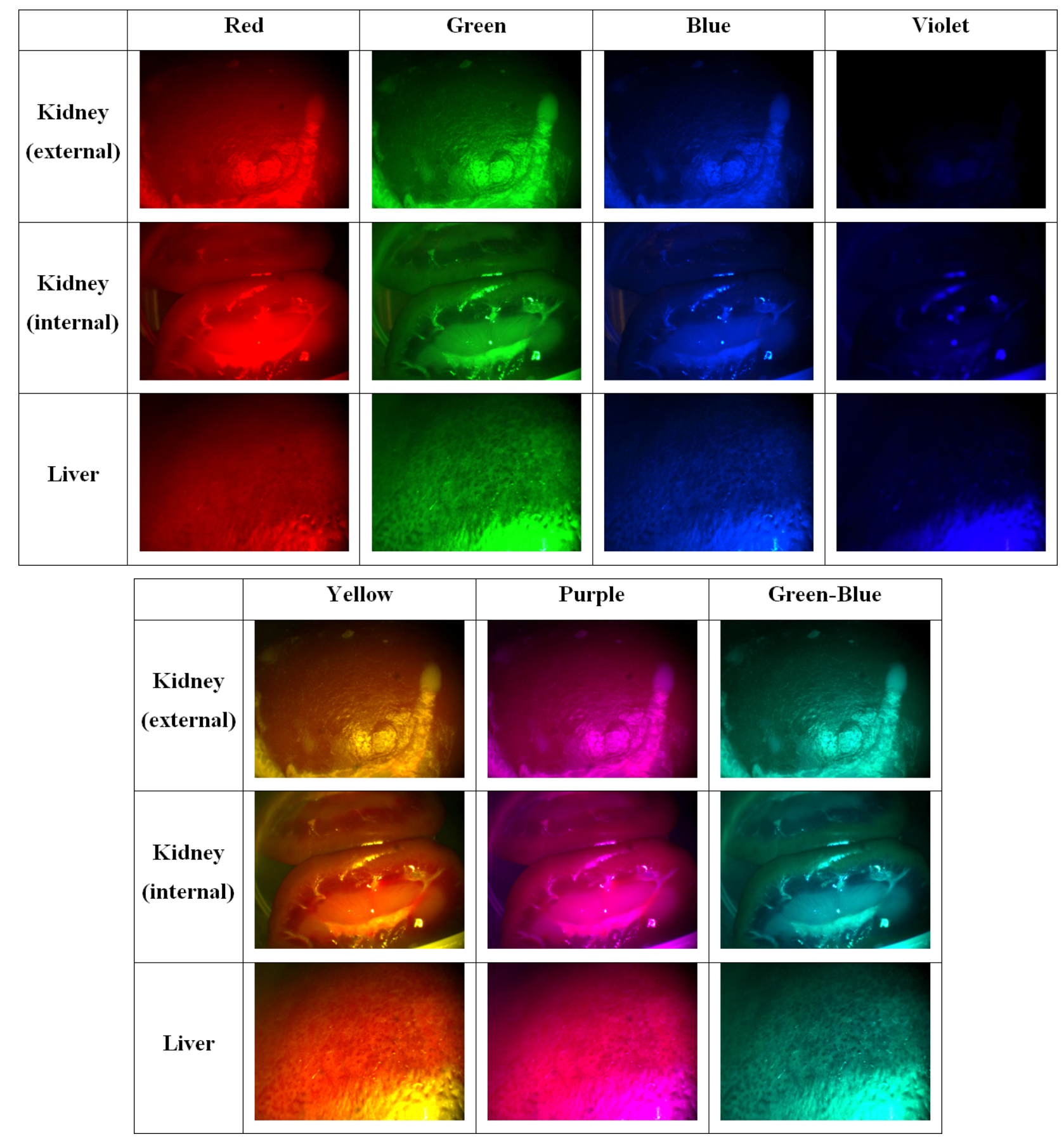

Figure 4. Colour balance variation in ex vivo tissue showing fat on the external surface of the kidney, the renal pyramids inside the kidney and the surface of the liver. LED settings used are defined in Table 1.

\subsection{In vivo trial}

Three anatomical sites were chosen to investigate laparoscopically during the porcine trial, including sections of the bowel and liver. These were imaged using the xenon lamp initially, followed by the LED source with varying colour settings as described in Section 3.2 above. The white light comparison is shown in Figure 5. As with the phantom and ex vivo comparisons detailed in Section 3.1, the images acquired using both sources were qualitatively very similar with slightly more red being apparent in the LED images. 


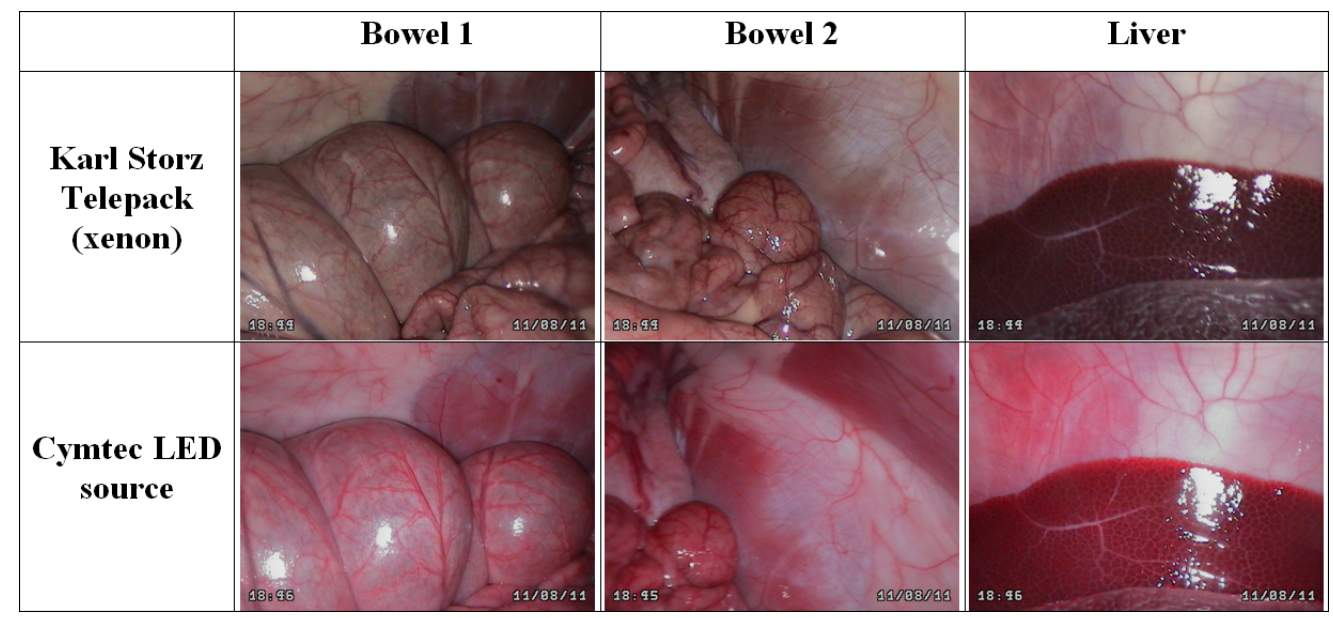

Figure 5. White light illumination of the abdominal cavity during a porcine laparoscopic procedure.

In narrowband imaging systems, illumination passband filters with a full-width at half-maximum (FWHM) of approximately $30 \mathrm{~nm}$ or less and centred on wavelengths in the green and blue range $\mathrm{e}^{3}$ are used to identify the superficial vasculature that is indicative of conditions such as oral neoplasia ${ }^{3}$, Barrett's oesophagus ${ }^{7}$ and urothelial cancer of the bladder $^{8}$. While the emission spectra of the LEDs in the device described here are too wide ( $>40 \mathrm{~nm}$ FWHM) to be considered 'narrow', their overlap with the camera's RGB filters does result in the creation of narrow bands, as shown in Figure 6 (a).
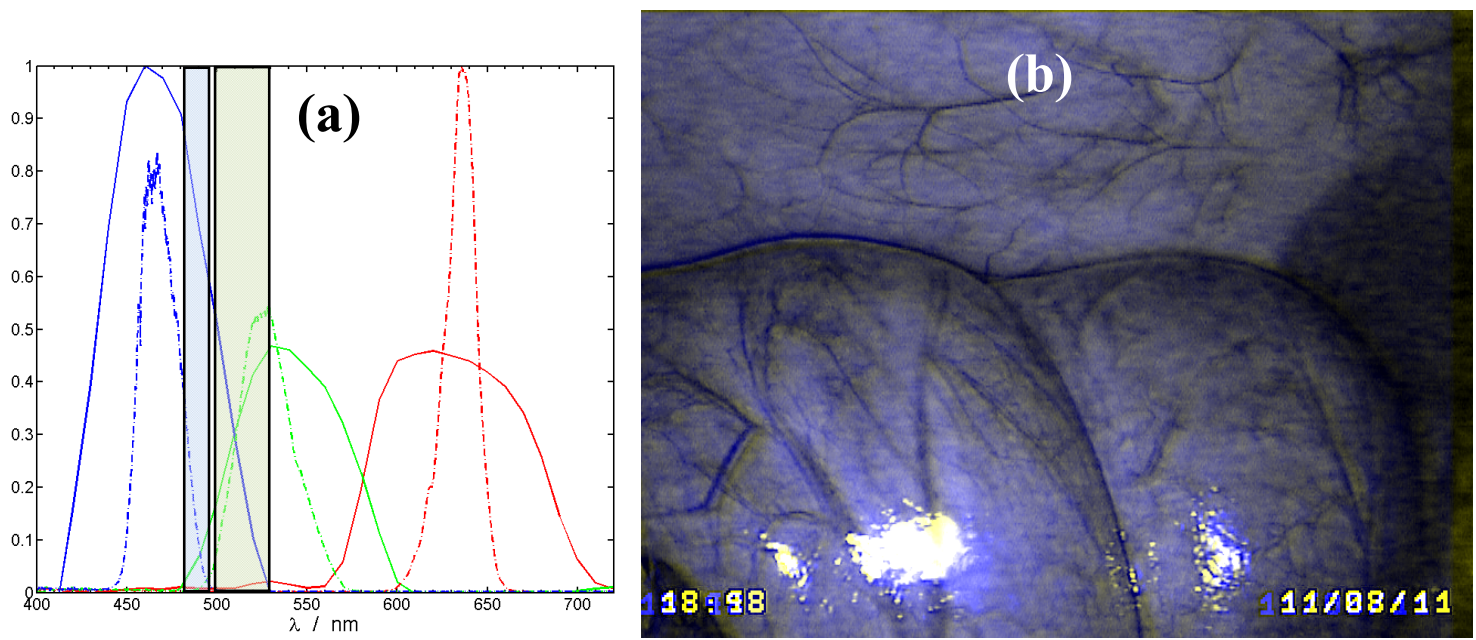

Figure 6. Narrowband imaging in vivo using the overlap between the LED emission spectra and camera RGB filter transmission spectra. (a) Narrow detection bands caused by the overlap of the LED emission spectra (dashed lines) and the camera's RGB filters (solid lines). (b) Narrowband image created using the detection bands shown in (a) emphasizing surface vasculature. Blurring is due to misalignment of the constituent images.

Figure 6 (b) shows a narrowband image generated using these overlapping regions. This was done by acquiring three images of the tissue in vivo using the green and blue LEDs in succession. The first detection band was obtained by taking the green colour plane from the blue-illuminated image, and the second was obtained by taking the blue colour plane from the green-illuminated image. These were then combined as the constituent colour planes of the 'RGB' image displayed in Figure 6 (b).

\section{DISCUSSION AND CONCLUSIONS}

An LED light source composed of four ultrabright LEDs that is compatible with endoscopic instruments has been presented. With the red, green and blue LEDs operating at full power, the device is capable of delivering $0.61 \mathrm{~W}$ at the distal end of an endoscope-specific fibre optic bundle. When used with a white-balanced camera, the output of the source 
provided qualitatively similar images to the standard xenon illuminant in imaging tests on tissue phantoms, ex vivo tissue samples and during an in vivo porcine laparoscopic procedure.

The spectral output of the light source may be tuned to any colour in a triangular region of the chromaticity diagram whose vertices are defined by the red, green and violet LEDs. This includes white light of varying colour temperature and can be adjusted to match a number of standard illuminants including daylight and xenon. Setting the emission colour of the light source to the blue-green region of the spectrum increases contrast in images of tissue by using light that has only penetrated superficially into the tissue (average penetration depth of less than $200 \mu^{3}$ ) and been strongly absorbed by blood.

For higher contrast data, a narrowband imaging regime was implemented by selecting the RGB colour planes that overlapped with the emission spectra of the blue and green LEDs in bands that were approximately 10 and $30 \mathrm{~nm}$ wide respectively. In this way, a narrowband feature can be obtained by simply plugging in the light source, unlike commercial systems, which use specialized filters on the imaging side. Enhancement of the surface vasculature was noted despite the presence of artefacts due to camera-tissue movement during the acquisition. This problem will be minimized in future iterations of the device by introducing computer-controlled light-switching to rapidly acquire the green and blue images.

Future development work will include improving coating design for higher luminous flux and the use of nextgeneration LEDs. Application testing of the device will see it used to image tissue with its violet LED in order to excite fluorophores such as ppIX for photodynamic diagnosis of bladder cancer.

\section{ACKNOWLEDGEMENTS}

Funding for this work was provided by NIHR grant II-3A-1109-10038 and ERC grant 242991. The authors are very grateful for the assistance of Northwick Park Institute for Medical Research (NPIMR), and Drs David Noonan and James Clark for their assistance with the in vivo measurements.

\section{REFERENCES}

[1] Clancy, N. T., Clark, J., Noonan, D. P., Yang, G.-Z., and Elson, D. S., "Light sources for single access surgery," Surg. Innov., 10.1177/1553350611421021 (2011).

[2] Babjuk, M., Soukup, V., Petrik, R., Jirsa, M., and Dvorácek, J., "5-aminolaevulinic acid-induced fluorescence cystoscopy during transurethral resection reduces the risk of recurrence in stage Ta/T1 bladder cancer," British Journal of Urology International, 96(6), 798-802 (2005).

[3] Takano, J. H., Yakushiji, T., Kamiyama, I., Nomura, T., Katakura, A., Takano, N., and Shibahara, T., "Detecting early oral cancer: narrowband imaging system observation of the oral mucosa microvasculature," Int. J. Oral Maxillofac. Surg., 39(3), 208-213 (2010).

[4] Lee, A. C. H., Elson, D. S., Neil, M. A., Kumar, S., Ling, B. W., Bello, F., and Hanna, G. B., "Solid-state semiconductors are better alternatives to arc-lamps for efficient and uniform illumination in minimal access surgery," Surg. Endosc., 23(3), 518-526 (2009).

[5] Smith, T., and Guild, J., "The C.I.E. colorimetric standards and their use," Trans. Opt. Soc., 33(3), 73-134 (1931).

[6] Clark, J., Södergren, M., Noonan, D., Darzi, A., and Yang, G.-Z., "The natural orifice simulated surgical environment (NOSsETM): exploring the challenges of NOTES without the animal model," J. Laparoendosc. Adv. S., 19(2), 211-214 (2009).

[7] Wolfsen, H. C., Crook, J. E., Krishna, M., Achem, S. R., Devault, K. R., Bouras, E. P., Loeb, D. S., Stark, M. E., Woodward, T. A., Hemminger, L. L., Cayer, F. K., and Wallace, M. B., "Prospective, controlled tandem endoscopy study of narrow band imaging for dysplasia detection in Barrett's esophagus," Gastroenterology, 135(1), 24-31 (2008).

[8] Bryan, R. T., Billingham, L. J., and Wallace, D. M. A., "Narrow-band imaging flexible cystoscopy in the detection of recurrent urothelial cancer of the bladder," Br. J. Urol., 101(6), 702-706 (2007). 